\title{
REVASCULARIZAÇÃO DO MIOCÁRDIO: DESVELANDO ESTRATÉGIAS DE REFERÊNCIA \\ E CONTRARREFERÊNCIA NA ATENÇÃO PRIMÁRIA À SAÚDE
}

\author{
MYOCARDIAL REVASCULARIZATION: UNVEILING \\ STRATEGIES IN REFERENCE AND COUNTER \\ REFERENCE IN PRIMARY HEALTH CARE
}

\section{REVASCULARIZACIÓN MIOCÁRDICA: DESCUBRIENDO ESTRATEGIAS DE REFERENCIA Y CONTRA REFERENCIA EN ATENCIÓN PRIMARIA DE SALUD}

\author{
Kamylla Santos da Cunha ${ }^{1}$ \\ Alacoque Lorenzini Erdmann ${ }^{2}$ \\ Giovana Dorneles Callegaro Higashi ${ }^{3}$ \\ Maria Aparecida Baggio ${ }^{2}$ \\ Carolina Kahl ${ }^{1}$ \\ Cintia Koerich ${ }^{3}$ \\ Betina Hörner Schlindwein Meirelles ${ }^{2}$ \\ Gabriela Marcellino de Melo Lanzoni ${ }^{2}$
}

\begin{abstract}
Objetivo: conhecer estratégias utilizadas por pacientes e profissionais de saúde no processo de referência e contrarreferência do paciente com indicação e submetido a cirurgia de revascularização miocárdica na Atenção Primária à Saúde (APS). Métodos: pesquisa qualitativa, ancorada na Teoria Fundamentada nos Dados. Resultados: os achados foram organizados em duas categorias: percebendo a Regulação como estratégia na articulação entre os níveis de atenção no processo de referência; desvelando ações estratégicas no acompanhamento do paciente submetido à Cirurgia de Revascularização Miocárdica na APS. Conclusões: as estratégias, no processo de referência, foram o Sistema de Regulação Nacional e o Tratamento Fora do Domicilio; na contrarreferência, a atuação da família, a busca ativa dos indivíduos pelos Agentes Comunitários de Saúde e a criação de grupos terapêuticos na APS. Essas estratégias, ainda fragilizadas, necessitam de articulação entre gestores e profissionais.
\end{abstract}

Descritores: Revascularização Miocárdica; Integralidade em Saúde; Referência e Consulta; Enfermeiros; Atenção Primária à Saúde.

Aim: to understand strategies used by patients and health professionals in the reference and counter reference process of the patient with indication and submitted to myocardial revascularization in Primary Health Care (PHC). Methods: a qualitative research, anchored by the Grounded Theory. Results: the findings were organized into two categories:

\footnotetext{
Enfermeiras. Mestrandas em Enfermagem. Universidade Federal de Santa Catarina. Florianópolis, Santa Catarina, Brasil. kamyllascunha@gmail.com; carolinakahl@hotmail.com

Enfermeiras. Doutoras em Enfermagem. Universidade Federal de Santa Catarina. Florianópolis, Santa Catarina, Brasil. alacoque.erdmann@ufsc.br; mariabaggio@yahoo.com.br; betina.hsm@ufsc.br; gabimrc@gmail.com

Enfermeiras. Doutorandas em Enfermagem. Universidade Federal de Santa Catarina. Florianópolis, Santa Catarina, Brasil. gio.enfermagem@gmail.com; cintia.koerich@ig.com.br
} 
Realizing the Regulation as a strategy for coordination between levels of care in the reference case; Unveiling strategic actions in the patient monitoring submitted to Myocardial Revascularization in PHC. Conclusions: the strategies, in the reference process, were the National Regulation System and Away from Home Care; in the counter reference, the role of the family, active search of the individuals by the Community Health Agents and the creation of therapeutic groups in the PHC. These still fragile strategies need articulation between management and professionals.

Descriptors: Myocardial Revascularization; Integrality in Health; Referral and Consultation; Nurses; Primary Health care.

Objetivo: conocer las estrategias utilizadas por los pacientes y profesionales de la salud en el proceso de referencia y contra referencia del paciente con indicación y sometido a una cirugía de revascularización coronaria en la atención primaria de salud (APS). Métodos: investigación cualitativa, anclado en la teoría fundamentada en los datos. Resultados: los resultados fueron organizados en dos categorias: darse cuenta de la regulación como una estrategia en la articulación entre los niveles de atención en el proceso de referencia; $y$ Revelando acciones estratégicas en la monitorización de pacientes sometidos a cirugía de revascularización coronaria en APS. Conclusiones: las estrategias, en el proceso de referencia, fueron el Sistema de Regulación Nacional y el Tratamiento Fuera del Domicilio; en la contra referencia, la actuación de la familia, la búsqueda activa de los individuos por Agentes Comunitarios de la Salud y la creación de grupos terapéuticos en la APS. Esas estrategias, aún fragilizadas, necesitan de articulación entre gestores y profesionales.

Descriptores: Revascularización Miocárdica; Integralidad em Salud; Remisión y Consulta; Enfermeros; Atención Primaria de Salud.

\section{Introdução}

É crescente o interesse dos gestores em saúde pelo perfil das doenças crônicas não transmissíveis, em especial das doenças cardiovasculares, as quais possuem elevada morbimortalidade no Brasil e no mundo ${ }^{(1)}$. Das complicações associadas às doenças cardiovasculares, o Infarto Agudo do Miocárdio (IAM) ganha destaque por representar a terceira maior causa de hospitalizações no Sistema Único de Saúde (SUS), gerando um custo aproximado de R \$ 1,9 bilhão ou 19\% dos gastos com internações por ano ${ }^{(2)}$.

A Cirurgia de Revascularização do Miocárdio (CRM), dentre outros, é um dos tratamentos indicados para IAM e tem como objetivo corrigir a isquemia miocárdica por obstrução das artérias coronárias. Esse procedimento pode oferecer aumento da expectativa de vida do paciente, assegurar o seu bem-estar biopsicossocial e o retorno breve às suas atividades cotidianas ${ }^{(3)}$. Devido à complexidade do processo pré e pós-operatório, é essencial a atenção criteriosa dos profissionais de saúde e de todos os envolvidos, observando o paciente de forma integral, bem como as suas particularidades, para buscar melhores resultados da experiência cirúrgica ${ }^{(4)}$.
A Atenção Primária à Saúde (APS) é porta de entrada preferencial para o sistema de saúde e realiza, no primeiro nível de atenção, ações individuais e coletivas voltadas à promoção, proteção e reabilitação da saúde e prevenção de $\operatorname{agravos}^{(5)}$. A assistência oferecida na APS proporciona à população o acesso aos diversos níveis de atenção à saúde por meio de estratégias que articulam as ações e os serviços necessários, proporcionando um atendimento inicial e continuado $^{(5,6)}$.

Estudo recente realizado em quatro grandes centros urbanos do Brasil com o objetivo de analisar desde a APS aos demais níveis de atenção à saúde, com o foco em integração entre níveis assistenciais, aponta como entrave a falta de integração entre diferentes níveis de atenção à saúde para a garantia do cuidado integral aos indivíduos $^{(7)}$. Nesse sentido, figura-se uma desarticulação entre os níveis de atenção à saúde no SUS, aspecto crítico a ser enfrentado na perspectiva de ampliar o acesso da população, particularmente ao paciente cardíaco, aos serviços oferecidos pelo sistema. 
O tratamento para o indivíduo com indicação de CRM e do indivíduo já submetido à cirurgia é complexo e necessita da comunicação e articulação entre os diferentes níveis de atenção à saúde, desde a APS até a atenção especializada, Unidades de Pronto Atendimento (UPAs), serviço de transporte emergencial e hospitais ${ }^{(8)}$.

O sistema de referência e contrarreferência é considerado uma ferramenta de gestão voltada à organização dos serviços de saúde, a fim de facilitar o acesso dos pacientes, inclusive os COronariopatas, aos níveis de maior complexidade, sustentado por critérios, fluxos e pactuações de funcionamento para assegurar a atenção integral aos indivíduos. Ao retornarem ao serviço de origem, esses sistemas possibilitarão à equipe o reconhecimento do acompanhamento recebido nos demais níveis de atenção, garantindo a efetividade do cuidado integral $^{(6,9)}$.

No entanto, outro estudo realizado no sul do Brasil, acerca da vivência de indivíduos que realizaram CRM, evidencia falhas de interação e ausência na estruturação do processo de referência e contrarreferência para indivíduo com indicação e submetido a CRM, o que reflete uma dificuldade na transição desses indivíduos entre os diferentes níveis de atenção à saúde no SUS ${ }^{(10)}$.

Assim, buscando respeitar as diretrizes e os princípios defendidos pelo SUS, torna-se necessário reconhecer e compreender o sistema de referência e contrarreferência disponibilizado ao indivíduo coronariopata que precisou ser submetido à CRM, bem como as estratégias utilizadas por eles e pelos profissionais de saúde para viabilizar o adequado atendimento/acompanhamento do paciente desde o diagnóstico da doença cardiovascular até o tratamento e a reabilitação.

Neste sentido, aponta-se como questionamento para este estudo: Quais as estratégias utilizadas por indivíduos e profissionais de saúde no processo de referência e contrarreferência do indivíduo com indicação e submetido à cirurgia de revascularização do miocárdio no cenário da APS? O estudo possui o objetivo de conhecer estratégias utilizadas por indivíduos e profissionais de saúde no processo de referência e contrarreferência do indivíduo com indicação e submetido à cirurgia de revascularização do miocárdio no cenário da APS.

\section{Métodos}

Trata-se de um estudo de abordagem qualitativa ancorado no referencial metodológico da Teoria Fundamentada nos Dados, que busca a compreensão do significado das relações e interações assentada nos dados com base na exploração dos fenômenos sociais. Na referida teoria, o processo de coleta e análise de dados é interdependente e ocorre de maneira simultânea, com o objetivo de identificar, desenvolver e relacionar conceitos, permitindo ao pesquisador maior aprofundamento do problema de pesquisa $^{(11)}$.

Os dados foram coletados no período de março a junho de 2014, em Florianópolis e Chapecó, municípios pertencentes ao estado de Santa Catarina, por meio de entrevistas individuais, abertas, registradas por gravação digital de voz. Participaram do estudo 41 pessoas (25 de Florianópolis e 16 de Chapecó), distribuídas em 3 grupos amostrais.

Para formar o primeiro grupo amostral, considerou-se como critério de inclusão: pacientes adultos de ambos os sexos submetidos a CRM há pelo menos seis meses, que já se encontravam em seu domicilio, identificados através da equipe de saúde da APS. Esse grupo foi constituído por 12 pacientes, sendo utilizada como pergunta inicial: Fale-me sobre seu acompanhamento de saúde na APS, antes e após a CRM? A fala dos pacientes enfatizava interface entre sua trajetória no processo de referência e contrarreferência e a atuação dos profissionais de saúde.

O segundo grupo amostral foi composto por profissionais que atendessem aos seguintes critérios: estar vinculado à rede de serviços da APS, bem como atuar na mesma unidade de saúde ou centro de especialidades há pelo menos seis meses. Este grupo foi formado por 7 agentes comunitários de saúde, 10 enfermeiros e 6 médicos. A questão norteadora para esse grupo foi: Como você vivencia o processo de referência e 
contrarreferência do indivíduo submetido à CRM no cenário da APS?

Diante do destaque realizado pelos profissionais em relação ao sistema de regulação e a necessidade de comunicação entre os níveis de atenção, buscou-se aprofundar algumas informações e alcançar maior consistência teórica com a formação do terceiro grupo amostral, composto por profissionais gestores vinculados ao Sistema de Regulação Municipal (SISREG) com no mínimo um ano de experiência na área. Assim, três médicos que trabalhavam no SISREG da Prefeitura Municipal de Florianópolis formaram esse último grupo amostral. A questão norteadora foi: Como a regulação municipal contribui para o processo de referência e contrarreferência do indivíduo submetido à CRM?

Destaca-se que os critérios de exclusão foram: pacientes menores de 18 anos e profissionais que estivessem afastados do trabalho, por quaisquer motivos, durante o período de coleta de dados.

Como a coleta e análise ocorrem de forma simultânea na Teoria Fundamentada nos Dados, a saturação teórica dos dados deu-se pela repetição de informações referentes ao fenômeno, ausência de novos elementos relevantes para a análise e consolidação das categorias e subcategorias em suas propriedades e dimensões.

A codificação é o processo central do desenvolvimento do modelo teórico, e ocorreu em três etapas que aconteceram simultaneamente: codificação aberta, axial e seletiva. Os códigos foram agrupados e seus agrupamentos deram origem às categorias e subcategorias, definidas e desenvolvidas em termos de suas propriedades e dimensões $^{(11)}$. O software NVIVO ${ }^{\circledR}$ foi utilizado para ordenação e organização dos dados no processo de codificação. O processo analítico foi construído segundo o Modelo Paradigmático, constituído por cinco componentes (contexto, causa, condição interveniente, estratégias e consequências) que explicam o fenômeno ${ }^{(11)}$.

O artigo é parte de um projeto maior intitulado "Paciente Cardíaco Revascularizado: Processo de Referência e Contrarreferência dos Serviços de Saúde de Santa Catarina”. Dada a importância e relevância do componente analítico Estratégia, optou-se por ampliar e aprofundar a discussão acerca deste, a fim de favorecer a compreensão do fenômeno.

Ressalta-se que a pesquisa atendeu aos aspectos éticos da Resolução n. 466/12 do Conselho Nacional de Saúde. O projeto foi submetido e aprovado pelo Comitê de Ética em Pesquisa com Seres Humanos da Universidade Federal de Santa Catarina (CEPSH/UFSC), protocolo número 120.184. Visando garantir o anonimato dos participantes, utilizou-se, para designá-los, a letra E seguida do número correspondente à ordem de realização das entrevistas (E1, E2, E3...) e da indicação do grupo amostral - primeiro grupo (G1), segundo grupo (G2), terceiro grupo (G3) - da seguinte forma: (E1G1); (E1G2); (E1G3)...

\section{Resultados}

Da análise dos dados do componente "Estratégia" emergiram duas categorias intituladas: "Percebendo a Regulação como estratégia na articulação entre os níveis de atenção no processo de referência" e "Desvelando ações estratégicas do acompanhamento do paciente submetido à Cirurgia de Revascularização do Miocárdio na Atenção Primária”. Essas categorias elucidam elementos e ferramentas específicas no caminho percorrido pelos indivíduos submetidos à CRM no SUS, além de seu fluxo e contrafluxo no sistema de saúde no estado de Santa Catarina.

A categoria "Percebendo a Regulação como estratégia na articulação entre os níveis de atenção no processo de referência" é composta por duas subcategorias. A primeira, intitulada "Referenciando o paciente para diferentes níveis de atenção à saúde", descreve os caminhos utilizados pelos pacientes de Florianópolis e de Chapecó na procura por atendimento em saúde, mediante sintomas de dor e desconforto torácico. Alguns participantes do estudo, ao apresentarem sinais e sintomas da doença cardíaca, procuraram, inicialmente, a APS em busca de atendimento, onde foram acolhidos e atendidos por profissionais de saúde. Mediante avaliação clínica e conforme necessidade houve solicitação 
médica de exames e/ou encaminhamento para consulta com especialistas em Cardiologia no nível secundário de atenção à saúde.

Os profissionais asseveraram que, ao ser detectada condição de urgência durante o atendimento ao paciente na APS, o Serviço de Atendimento Móvel de Urgência (SAMU) é acionado, visto que possui equipamentos e profissionais capacitados para realizar o atendimento e o transporte seguro até o hospital de referência. No entanto, no estudo, não houve relatos que indicassem o uso do SAMU para atendimento e remoção de pacientes coronariopatas em condição de urgência para o nível terciário de saúde.

Normalmente o paciente chega aqui na Atenção Básica reclamando de dor forte e desconforto. Ele é atendido no acolhimento e, depois, conforme necessidade, encaminhado para consulta. (E14G2).

Os pacientes dão entrada na atenção básica, são atendidos e aqueles que necessitam de especialistas são agendados para a atenção secundária, que, aqui em Chapecó é o CRESM, um centro de referência especializada. (E28G2).

Em Florianópolis, os relatos apontam que, após as consultas agendadas por intermédio das Unidades Básicas de Saúde (UBS), os pacientes são encaminhados para atendimento especializado em Policlínicas ou UPAs. Após serem avaliados pelo Cardiologista e identificadas as necessidades de procedimentos invasivos para diagnóstico ou necessidade da realização de cirurgia cardíaca, são inseridos no Sistema de Regulação Municipal e regulados para os hospitais de Cardiologia.

Se o paciente realmente houver indicação clínica, nós encaminhamos para os cardiologistas da atenção secundária, nas policlinicas ou unidades de pronto atendimento. (E14G2).

Em Chapecó, esse processo ocorre de forma quase semelhante ao de Florianópolis. Porém os participantes apontam o Centro de Referência em Saúde Municipal (CRESM) como o nível secundário de atenção à saúde, para consulta com o especialista em Cardiologia. As consultas são agendadas por intermédio das UBS.
No CRESM, os pacientes, ao serem avaliados pelo Cardiologista e identificados com doença coronária aterosclerótica, que requer realização de procedimentos invasivos para diagnóstico da extensão da doença e/ou tratamento da obstrução coronária, bem como para a realização de cirurgia cardíaca, são encaminhados para o hospital de referência em Cardiologia mais próximo, situado em Xanxerê, ou para outros hospitais de referência do estado de Santa Catarina.

Os pacientes chegam aqui no CRESM, na maior parte das vezes, encaminhados da rede básica [...] nós atendemos e se verificamos a necessidade de alguma complementação com exames ou um procedimento cirúrgico, nós encaminhamos para nossa referência regional, que é Xanxerê ou para nossa referência estadual, Florianópolis. (E30G2).

As especialidades são todas agendadas para o CRESM, local de referência para realizar as consultas com o cardiologista [...] Após avaliação clinica, se houver necessidade, os pacientes são encaminhados para hospital de referência em Xanxerê. (E26G2).

Na segunda subcategoria, intitulada "Considerando a Regulação como estratégia no processo de referência”, os participantes de Florianópolis destacaram o Sistema de Regulação Municipal e Estadual (SISREG) como principal estratégia na referência do paciente da Atenção Primária a Saúde para assistência nos serviços na média complexidade. Esse sistema regula as consultas com especialistas e exames específicos e, na alta complexidade, regula os leitos para internação hospitalar no hospital de referência. É responsável ainda pela regulação das unidades de saúde de Florianópolis, onde o paciente é atendido pela equipe, sendo verificada a necessidade de atenção especializada, e suas informações de saúde são inseridas no sistema e verificadas por um profissional da regulação para avaliar e classificar o risco. Por fim, o paciente é encaminhado para o devido nível de atenção. Para tanto, é imprescindível que as informações contidas no sistema sejam preenchidas adequadamente, conforme mostra a fala a seguir: 
O funcionário que insere as informações do paciente no SISREG deve ter um treinamento; é responsável por passar a situação clinica, escolher para qual serviço ele está referenciando aquele paciente [...] as informações clinicas devem ser preenchidas corretamente lá na unidade, que, aí, o regulador vai abrir (no sistema informatizado) $e$ verificar o que foi escrito para realizar o julgamento, se existe a necessidade, ou não, de encaminhar o pedido que está sendo requerido. (E35G3).

Os participantes de Chapecó destacaram o Tratamento Fora do Domicílio (TFD) como estratégia utilizada pelos profissionais de saúde, o qual permite que pacientes de Chapecó, com coronariopatia, possam ser referenciados para outros municípios, a fim de realizarem consultas, exames e/ou tratamento especializados e/ou de alta complexidade não disponíveis nesse município.

Quando o paciente precisa fazer algum exame que nós não temos aqui em Chapecó, nós encaminhamos ele para o setor de [Tratamento Fora do Domicílio] TFD. Ele que vai encaminhar o paciente para outro município para receber atendimento. (E28G2).

A categoria "Desvelando ações estratégicas do acompanhamento do paciente submetido à Cirurgia de Revascularização do Miocárdio na Atenção Primária” é composta por duas subcategorias. A primeira delas é intitulada "Destacando a participação de familiares e dos Agentes Comunitários de Saúde (ACS) como estratégias no processo de contrarreferência”. Nesta destaca-se falha de comunicação entre os profissionais de saúde do nível hospitalar com os profissionais de saúde da APS na contrarreferência dos indivíduos que retornam ao seu domicílio após CRM, com necessidade de múltiplos cuidados pelos profissionais com vistas à reabilitação cardíaca. Os relatos evidenciaram a inexistência da comunicação verbal, via sistema ou documento de alta hospitalar, para entregar aos profissionais de saúde da APS. Os profissionais da APS, por meio de informações dos familiares ou da visita de rotina dos ACS, passam a ter conhecimento de que um indivíduo em sua área de abrangência já recebeu alta hospitalar e está em recuperação no seu domicílio.

No primeiro momento, a família vai até a APS e/ou solicita ao ACS visita domiciliar da equipe de saúde ao indivíduo em seu domicílio. Na segunda situação, mediante a visita domiciliar de rotina, os ACS identificam algum paciente domiciliado na sua área de abrangência que foi submetido à CRM. Em ambas as situações, os ACS estão envolvidos na identificação desses indivíduos e, ao receberem essas informações, comunicam ao enfermeiro que, junto com o técnico de enfermagem, realiza a primeira avaliação da saúde do paciente. Os familiares ressaltaram a importância do trabalho dos ACS no desempenho de suas atribuições junto à comunidade, conforme evidenciam as falas:

\footnotetext{
Eu gosto de receber a visita dos agentes comunitários. Eles são muito atenciosos sempre e perguntam como eu estou e depois disso eu recebia a visita dos profissionais de saúde. (E5G1).
}

Os agentes comunitários geralmente são bem competentes e bem interessados sobre o seu serviço, sendo as pessoas mais indicadas para fazer a busca ativa. (E29G2).

Na segunda subcategoria, intitulada "Identificando a necessidade de criação de grupos terapêuticos para acompanhamento dos pacientes submetidos à Cirurgia de Revascularização Miocárdica”, os participantes ressaltaram que, diante das fragilidades da contrarreferência, os grupos terapêuticos podem proporcionar um espaço para as orientações referentes à qualidade da recuperação do indivíduo revascularizado, conforme fala a seguir:

Não existe nenhum grupo terapêutico na unidade para pacientes ou familiares de pacientes que fizeram a cirurgia de revascularização [...] Acredito que se houvessem grupos terapêuticos ajudaria muito, porque garantiriam mais informações aos pacientes, não se limitando a número de pessoas, $e$ assim maior troca de informações e experiências, onde os paciente vão ficando mais tranquilizados. (E5G1). 
A criação de grupos terapêuticos na APS voltados ao paciente submetido a CRM seria uma importante estratégia na contrarreferência, visto que, de acordo com os relatos, possibilitaria maior acompanhamento desse paciente, para além das orientações de cuidado pós-cirúrgicos, estabelecendo vínculo com a APS, mas também proporcionando um momento de troca entre pacientes na mesma situação de recuperação.

\section{Discussão}

As redes de atenção à saúde, conforme determina o SUS, priorizam condições estruturalmente adequadas à oferta de serviços que se comuniquem entre si de forma horizontalizada em diferentes fluxos para a qualidade e efetivação do cuidado integral ${ }^{(12)}$. O acompanhamento do paciente com indicação e submetido à cirurgia de revascularização do miocárdio, no processo de referência e contrarreferência, necessita da articulação de todos os níveis da rede de atenção à saúde, como o primário, secundário, terciário e os serviços de apoio, visando a oferta de uma atenção contínua e integral.

Os achados deste estudo evidenciam que a APS em Florianópolis conta com o apoio do SISREG nacional, na organização do fluxo de atenção à saúde e referência dos pacientes nos diferentes níveis de complexidade. Desenvolvido e disponibilizado pelo Ministério da Saúde (MS), esse é um software com sistema informatizado, on-line e gratuito, que funciona como ferramenta promotora de equidade, acessibilidade e integralidade, planejando e organizando o fluxo dos pacientes, visando à humanização dos serviços, diminuição do tempo de espera, controle de fluxo e otimização na utilização dos recursos, a fim de melhorar o acesso aos serviços em todos os níveis ${ }^{(13)}$.

Estudo bibliográfico realizado com o objetivo de identificar, na literatura, produções relacionadas ao funcionamento do SISREG aponta fatores que dificultam o acesso ao sistema, como a não priorização de agendamento de consultas pelos próprios profissionais que trabalham na marcação e que manipulam o software ${ }^{(14)}$.
De modo semelhante, nesta pesquisa, os relatos elucidaram que, para ter acesso ao sistema e alimentá-lo com as informações pertinentes ao processo de referenciamento dos pacientes, os profissionais precisam passar por um treinamento. O MS preconiza a realização de treinamento dirigido aos profissionais que utilizam o sistema, para conhecerem o escopo de funcionalidades que compõe uma central de regulação e entenderem os critérios das condições que os reguladores avaliam. Uma solicitação bem referenciada necessita ter uma justificativa clara, para que seja consumada. Além disso, deve seguir um protocolo clínico que justifique a sua importância para funcionar de maneira rápida e prática ${ }^{(13)}$.

Paralelamente ao SISREG, em Chapecó, o processo de referência dos pacientes submetidos a CRM conta ainda com o apoio do setor de TFD. Instituído pelo MS por meio da Portaria SAS/MS n. 055/99, o TFD não é um sistema informatizado como o SISREG, mas tem o objetivo essencial de garantir ao paciente o acesso a serviços assistência em outros municípios e/ou estados, quando esgotadas as formas de tratamento de saúde na localidade em que reside. Assim, o TFD, além de garantir acesso a esses serviços de continuidade, também é responsável por oferecer condições para o deslocamento e a permanência do paciente e seu acompanhamento fora do seu domicílio ${ }^{(15)}$. A fim de garantir, sem rompimento do fluxo assistencial, a continuidade do cuidado ao paciente que necessita de exames especializados ou intervenção cirúrgica, o setor possibilita o tratamento do paciente em serviços de saúde mais próximos de sua localidade.

Sendo a APS considerada o centro de COmunicação entre os níveis de atenção à saúde, ordenando os fluxos e contrafluxos dos níveis primário, secundário e terciário, após o paciente ser submetido a CRM e receber alta hospitalar, é contrarreferenciado para a APS. No entanto, os dados mostraram que o processo de contrarreferência não ocorre de forma tão linear como o processo de referência. Os relatos sustentaram que, após o paciente receber alta hospitalar e retornar para a sua casa, depara-se com entraves, como a falta de comunicação entre a instituição 
hospitalar e a APS. Corrobora o exposto o estudo realizado com profissionais da ESF sobre o sistema de referência e contrarreferência, no contexto do SUS, ao apontar que 59,1\% dos entrevistados afirmaram que os usuários dos serviços de saúde não são contrarreferenciados para a APS após o atendimento na alta complexidade, sugerindo um modelo fragmentado de atenção à saúde e falha de comunicação entre os níveis de atenção ${ }^{(6)}$.

Dessa forma, para não comprometer as ações de cuidado dispensadas ao indivíduo submetido a CRM na alta hospitalar, os relatos deste estudo apontam os familiares e a busca ativa dos pacientes pelos profissionais da APS como estratégia de resgate na contrarreferência. $\mathrm{O}$ apoio da família é valorizado para dar continuidade aos cuidados dos indivíduos submetidos a CRM, sendo os familiares essenciais para facilitar o manejo dos novos enfrentamentos e promover adequações às alterações e às possíveis dificuldades ${ }^{(16)}$.

Um estudo que buscou conhecer as expectativas de pacientes submetidos à cirurgia de revascularização do miocárdio no momento da alta hospitalar evidenciou que as expectativas apontadas foram relacionadas ao processo de recuperação e retorno às atividades diárias. O período de alta hospitalar mostra-se desafiador para o indivíduo e seu núcleo familiar, sobressaindo-se sentimentos de ansiedade e preocupação com o futuro, atrelado, muitas vezes, ao déficit de conhecimento relacionado às mudanças no estilo de vida impostas pelo processo de adoecimento. A cirurgia provoca um impacto significativo na vida dos pacientes, de modo que a intervenção efetiva da enfermagem pode implicar positivamente tanto na recuperação e reabilitação do paciente revascularizado quanto no fortalecimento de sua rede de apoio ${ }^{(17)}$.

De acordo com os depoimentos dos participantes deste estudo, os familiares também se configuram como estratégias que favorecem e fortalecem o processo de contrarreferência, amparando o paciente em suas necessidades e oferecendo apoio e suporte emocional, social, espiritual e financeiro. Nesta perspectiva, estudo sobre manifestações psicológicas do paciente no pré e pós-operatório da cirurgia cardíaca apontou que as principais as manifestações psicológicas apresentadas pelo estudo foram: ansiedade, depressão, medo, vivências traumáticas, alteração no padrão de sexualidade e distúrbio no padrão de sono. Reconhecer como o paciente enfrenta e lida com o processo cirúrgico e o seu retorno ao seio familiar é um aspecto importante tanto para os profissionais quanto para os familiares que o acompanham ${ }^{(18)}$. O componente familiar também assume importância como apoio/ amparo ao paciente desde o início da descoberta da doença até o enfrentamento das mudanças em seu novo contexto de vida acarretado pela doença.

Estudo que objetivou identificar as estratégias utilizadas pelos ACS em sua rede de relações e interações para melhoria da assistência à saúde e suas repercussões em um centro de saúde trouxeram em seus resultados que a equipe de saúde considera o trabalho dos ACS uma importante ferramenta para alcance dos usuários e revela que esses são considerados a melhor forma para se comunicar com a comunidade, pois, com base no conhecimento dos problemas dos usuários, o ACS identifica a necessidade de informar à equipe multiprofissional, facilitando-lhe o contato com o indivíduo que necessita de cuidados ${ }^{(19)}$.

Os depoimentos dos participantes evidenciaram que os ACS são estratégias que favorecem o contato e o encontro com o paciente após o evento cirúrgico e o retorno ao seu lar, identificando, assim, suas demandas e necessidades físicas, sociais, biológicas e emocionais. No entanto, torna-se necessário que o ACS, em seu contexto laboral, utilize ferramentas que superem o conhecimento apoiado no modelo biomédico vinculado à doença, isto é, seja detentor de habilidades que possibilitem atuar no âmbito social de cada família e no campo das necessidades de saúde de cada usuário, em prol de um modelo de atenção que promova a integração do trabalho em equipe ${ }^{(20)}$.

Assim como os ACS, os grupos terapêuticos também foram considerados pelos participantes do estudo como estratégias que impulsionam o processo de contrarreferência, com ênfase 
na criação de grupos terapêuticos ou de novos espaços relacionais que atendam às necessidades específicas do paciente submetido a CRM. Estudo realizado em Uberaba (MG), para conhecer a percepção de enfermeiros da ESF sobre a educação em saúde, aponta que os profissionais de saúde possuem uma próxima relação com grupos terapêuticos, mas ressaltam a verticalização desta prática e a necessidade de reflexão a respeito desta abordagem para valorização do saber popular e interações dialógicas para a concretização de vínculo com o usuário ${ }^{(21)}$.

Outro estudo sobre o impacto da qualidade de vida após o procedimento cirúrgico, envolvendo dois grupos de pacientes - o primeiro constituído por pós-operatórios que participavam do programa de reabilitação ou realizavam alguma atividade física regularmente; e o segundo composto por um grupo de pacientes que não participavam do programa de reabilitação e não praticavam nenhuma atividade física - revelou que, após a realização da cirurgia, houve piora na qualidade de vida dos pacientes do segundo grupo. Por outro lado, em relação aos que participavam do programa e faziam atividade física, constatou-se melhora ${ }^{(22)}$.

Nessa perspectiva, os participantes desta pesquisa entendem a ausência de grupos terapêuticos como uma lacuna para a vida e a recuperação dos pacientes submetidos à CRM. Além disso, ressaltaram que a criação desses grupos poderia estabelecer um vínculo entre o paciente e os profissionais de saúde da APS, além da socialização de vivências e experiências entre os pacientes, fortalecendo a sua reabilitação cardíaca.

\section{Considerações finais}

O estudo revelou estratégias de referência e contrarreferência utilizadas no cenário da APS para indivíduos submetidos a CRM, evidenciando, no processo de referência, o SISREG e o setor de TFD como ferramentas de direcionamento dos indivíduos para outros níveis de atenção à saúde, que ofereçam serviços especializados para confirmação do diagnóstico da doença cardíaca, até serviços de alta complexidade para a realização da CRM.

No processo de contrarreferência, após alta hospitalar, apesar dos entraves devido à falha de comunicação entre instituição hospitalar e APS, fragilizando a continuidade de atenção a esses indivíduos, os resultados evidenciaram estratégias que fortalecem a contrarreferência, como a atuação da família e a busca ativa dos indivíduos realizada pelos ACS orientados pela equipe multiprofissional, assim como a criação de grupos terapêuticos na APS para auxílio no cuidado desses indivíduos, os quais enfrentam diversos sentimentos e emoções, medos, angústias e inseguranças relacionadas a um momento de mudança no estilo de vida após a CRM.

Diante desse cenário, vislumbra-se o desafio dos profissionais da APS para garantir o atendimento do indivíduo submetido à CRM em todas as suas dimensões, considerando a existência de um processo de referência e contrarreferência ainda fragilizado. Para isso, é imprescindível interesses articulados entre gestores e profissionais que prestam atendimento direto, a fim de fortalecer o vínculo entre os níveis de atenção à saúde no SUS garantindo a integralidade do cuidado à saúde desses indivíduos.

\section{Fonte de Financiamento}

Este estudo recebeu suporte financeiro da Fundação de Amparo a Pesquisa e Inovação do Estado de Santa Catarina (FAPESC) por meio processo n. 1459/2012.

\section{Referências}

1. Ducan BB, Chor D, Aquino EML, Bensenor IM, Mill JG, Schmidt MI, et al. Doenças crônicas não transmissíveis no Brasil: prioridade para enfrentamento e investigação. Rev Saúde Pública. 2012;46(Supl):126-34.

2. Ministério da Saúde (BR). Datasus. Sistema de informações hospitalares. Internações e valor total de internações segundo capítulo CID-10 [Internet]. Brasília (DF); 2011. [citado 2011 out 12]. Disponível em: http://www.datasus.gov.br 
3. Gomes WJ. Cirurgia de revascularização miocárdica com e sem circulação extracorpórea. O cirurgião cardíaco deve dominar ambas as técnicas. Rev bras cir cardiovasc. 2012;27(2):v-viii.

4. Borges DL, Nina VJS, Lima RO. Características clínicas e demográficas de pacientes submetidos à revascularização do miocárdio em um hospital universitário. Rev pesq saúde. 2013;14(3):171-4.

5. Almeida PF, Fausto MCR, Giovanella L. Fortalecimento da atenção primária à saúde: estratégia para potencializar a coordenação dos cuidados. Rev Panam Salud Publica. 2011;29(2):84-95

6. Costa SM, Ferreira A, Xavier LR, Guerra PNS, Rodrigues CAQ. Referência e contrarreferência na saúde da família: percepções dos profissionais de saúde. Rev APS. 2013;16(3):287-93.

7. Almeida PF, Giovanella L, Mendonça MHM, Escorel S. Desafios à coordenação dos cuidados em saúde: estratégias de integração entre níveis assistências em grandes centros urbanos. Cad Saúde Publica. 2010:26(2):286-98

8. Marcolino MS, Brant LCC, Araújo JG, Nascimento BR, Castro LRA, Martins P, et al. Implantação da linha de cuidado do infarto agudo do miocárdio no município de Belo Horizonte. Arq bras cardiol. 2013;100(4):307-14.

9. Corrêa ÁCP, Dói HY. Contrarreferência de mulheres que vivenciaram gestação de risco a unidades de saúde da família em Cuiabá. Rev ciênc cuid saúde. 2014;13(1):104-10.

10. Erdmann AL, Lanzoni GMM, Callegaro GD, Baggio MA, Koerich C. Compreendendo o processo de viver significado por pacientes submetidos a cirurgia de revascularização do miocárdio. Rev latino-am enferm. 2013;21(1):[8 telas].

11. Strauss A, Corbin J. Pesquisa qualitativa: técnicas e procedimentos para o desenvolvimento de teoria fundamentada. Tradução Luciane de Oliveira da Rocha. $2^{\underline{a}}$ ed. Porto Alegre: Artmed; 2008.

12. Silva SF. Organização de redes regionalizadas e integradas de atenção à saúde: desafios do Sistema Único de Saúde (Brasil). Ciênc Saúde Coletiva. 2011;16(6):2753-62.

13. Ministério da Saúde. Conselho Nacional de Saúde (BR). Manual de implantação de complexos reguladores. Brasília (DF); 2006.
14. Pinto JR, Carneiro MGD. Avaliação do agendamento online de consultas médicas especializadas através da central de regulação do SUS. Saúde Coletiva. 2012;58(1):123-8.

15. Secretaria da Saúde (SC). Superintendência de Serviços Especializados e Regulação. Gerência dos Complexos Reguladores. Manual de normatização do Tratamento Fora do Domicílio - TFD no Estado de Santa Catarina. Florianópolis (SC); 2013.

16. Garcia RP, Budó MLD, Simon BS, Wünsch S, Oliveira SG, Barbosa MS. Vivências da família após infarto agudo do miocárdio. Rev Gaúcha Enferm. 2013;34(3):171-8.

17. Cielo C, Silveira M, Arboit EL. Expectativas de pacientes submetidos à cirurgia de revascularização do miocárdio no momento da alta hospitalar. Fundam care [online] 2015 [citado 2016 mar 4];7(3):2670-87. Disponível em: http://www.seer. unirio.br/index.php/ cuidadofundamental/article/ view/3623.

18. Quintana JF, Kalil RAK. Cirurgia cardíaca: manifestações psicológicas do paciente no pré e pós-operatório. Psicol hosp. 2012:10(2):17-32.

19. Lanzoni GMM, Cechinel C, Meirelles BHS. Agente Comunitário de Saúde: estratégias e consequências da sua rede de relações e interações. Rev Rene. 2014;15(1);123-31.

20. Galavote HS, Prado TN, Maciel ELN, Lima RCD. Desvendando os processos de trabalho do agente comunitário de saúde nos cenários revelados na Estratégia Saúde da Família no município de Vitória (ES, Brasil). Ciênc saúde coletiva. 2011;16(1):231-40.

21. Cervera DPP, Parreira BDM, Goulart BF. Educação em saúde: percepção dos enfermeiros da atenção básica em Uberaba (MG). Ciênc saúde coletiva. 2011;16(SupL 1):1547-54.

22. Sampaio JKVR, Figueiredo Neto JA, Queiroz LLC, Sousa RML, Reis LMCB, Silva FDMAM. Impacto na qualidade de vida pós-angioplastia coronariana ou revascularização do miocárdio. Rev bras cardiol. 2013:26(5):337-46.

Artigo apresentado em: 12/2/2016

Aprovado em: 17/3/2016

Versão final apresentada em: 18/3/2016 